\title{
From Pressure-Volume Relationship to Volume-Energy Relationship: A Thermo-Statistical Model for Alveolar Micromechanics
}

\author{
Kyongyob Min \\ Ueda-Shimotanabe Hospital, Noborimachi, Takatsuki, Osaka, Japan \\ Email: in1007@osaka-med.ac.jp
}

How to cite this paper: Min, K. (2020) From Pressure-Volume Relationship to Volume-Energy Relationship: A Thermo-Statistical Model for Alveolar Micromechanics. Applied Mathematics, 11, 11161131.

https://doi.org/10.4236/am.2020.1111075

Received: September 27, 2020

Accepted: November 10, 2020

Published: November 13, 2020

Copyright $\odot 2020$ by author(s) and Scientific Research Publishing Inc. This work is licensed under the Creative Commons Attribution International License (CC BY 4.0).

http://creativecommons.org/licenses/by/4.0/

(c) (i) Open Access

\begin{abstract}
The connective tissue fiber system and the surfactant system are essential and interdependent components of lung elasticity. Despite considerable efforts over the last decades, we are still far from understanding the quantitative roles of either the connective tissue fiber or the surfactant systems. Through thermo-statistic considerations of alveolar micromechanics, the author introduced a thermo-statistic state function "entropy" to analyze the elastic property of pulmonary parenchyma based on the origami model of alveolar polyhedron. By use of the entropy for alveolar micromechanics, from the logistic equation for the static pressure $(P)$-volume $(V)$ curves including parameters $a, b, c$, and $k(V-a=b /[1+\exp \{-k(P-c)\}])$, a set of equations was obtained to define the internal energy of lungs $\left(U_{L}\right)$ and its corresponding lung volume $\left(V_{L}\right)$. Then, by use of parameters $a, b, c$, and $k$, an individual volume-internal energy $\left(V_{L}-U_{L}\right)$ diagram was constructed from reported data in patients on mechanical ventilation. Each $V_{L}-U_{L}$ diagram constructed was discussed that its minimal value $U_{o}=c(a+b / 2)$ and its shape parameter $b / k$ represent quantitatively the energy of tissue force and the energy of surface force. Furthermore, by use of the $V_{L}-U_{L}$ relationship, the hysteresis of lungs estimated by entropy production was discussed as dependent on the difference in the number of contributing pulmonary lobules. That is, entropy production might be a novel quantitative indicator to estimate the dynamics of the bronchial tree. These values obtained by combinations of parameters of the logistic $\mathrm{P}-\mathrm{V}$ curve seem useful indicators to optimize setting a mechanical ventilator. Thus, it is necessary to develop easy tools for fitting the individual sigmoid pressure-volume curve measured in the intensive care unit to the logistic equation.
\end{abstract}




\section{Keywords}

Thermo-Statistic Entropy, Thermodynamic Temperature, Sigmoid

Pressure-Volume Curve, Logistic Equation, Volume-Energy Relationship

\section{Introduction}

Measuring pressure-volume (PV) curves have been used in setting mechanical ventilation to quantify the elastic properties of lungs. Classical physiology has explained that the elastic property originates from the basic components of the lung skeleton: 1) a continuous network of elastic fibers in the alveolar walls that connects arteries, airways, and interstitial space (the tissue force), and 2) the force generated at the surface between the air and the alveolar surfactant lining layer (the surface force) [1]. The quasi-static PV curve of the respiratory system describes the mechanical behavior of the lung parenchyma during inflation and deflation, and measuring PV curves has been used in research to quantify the elastic properties of lung parenchyma [2]. Despite considerable efforts over the last decades, we are far from understanding the quantitative indicators of either the connective tissue fiber or the surfactant systems for optimization of mechanical ventilation.

Elasticity is measured in the excised lung as the relationship between lung volume and pressure measured at the closed airway (PV curve). Experimental study revealed, as shown in Figure 1, that the PV curve of an excised lung filled with air (dashed lines) is quite different from that of a degassed lung refilled with saline solution (solid lines) [1] [2]. It has been recognized that the curve of the saline-filled lung mainly represents the properties of the tissue components whereas that of the gas-filled lung includes both the tissue properties and the surface tension exerted along the alveolar walls. During inflation, the work done

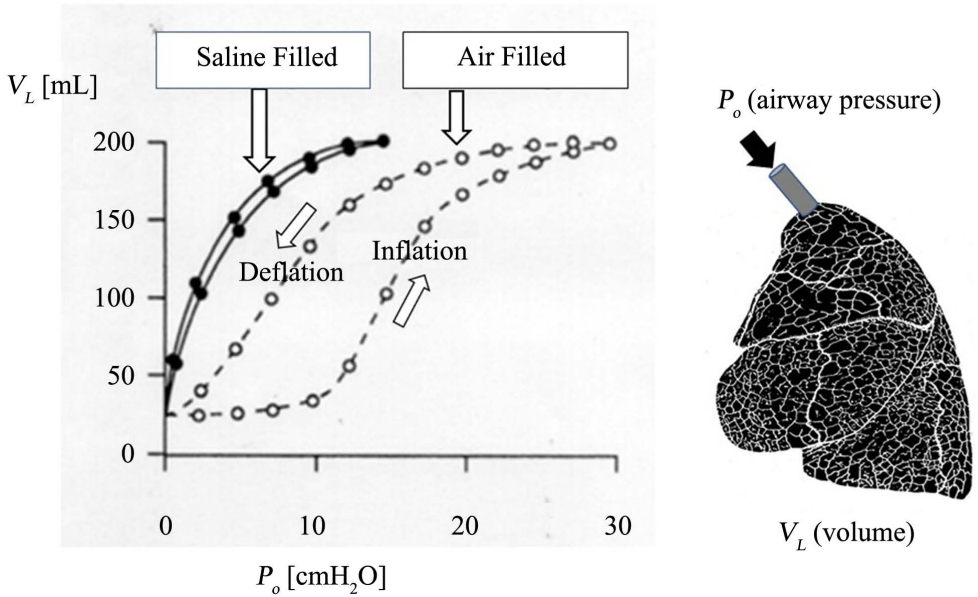

Figure 1. Pressure-Volume (PV) curves. Relationship between lung volume $\left(V_{L}\right)$ and airway pressure measured at the closed airway $\left(P_{o}\right)$. The PV curves of an excised lung filled with air (dashed lines) and with saline (solid lines). 
on the lung components is mainly performed on the elastic network and on the interface between gas and the lining layer, both of which increase in tension. The energy is partially recovered, partially the energy stored in the fibrous network during deflation. The area of the loop circumscribed by the line of inflation-deflation is called hysteresis, which is explained as energy dissipation at the alveolar surface. Fredberg and Kamm calculated that during a lifetime the alveolar structures must cope with breathing related alveolar strain for up to $10^{9}$ strain cycles, and considered these strains as extreme and would appear to call for specialized tissue structures from the standards of common engineering materials [3].

More than 30 years ago, Gil et al. showed that using quasi-static conditions and vascular perfusion fixation of lung tissue at defined inspiratory and expiratory pressures revealed that different mechanisms of septal wall deformation are involved as follows: 1) recruitment/derecruitment of alveolar units; 2) isotropic stretching and destretching with balloon-like changes of alveolar size; 3) changes in alveolar shape (e.g. from dodecahedral to spherical and vice versa), which, due to different geometry, are linked with changes in alveolar size; and 4) folding and unfolding of alveolar walls and accordion-like deformation that might resemble the folding and unfolding of a paper bag [4] [5]. Kitaoka et al. developed the folding and unfolding of paper bags for the "origami model" of alveolar polyhedron [6], by use of which Min explained the elastic properties of lungs as entropy change of alveolar micromechanics [7]. The entropy change of alveolar microstructure is less in dissipating energy than the stretching change of tissue as well.

Reports have indicated that PV curves are fit to the exponential equation such as $V=A-B \exp (-K P)$, where $V$ is volume, $P$ is transpulmonary pressure, and $A, B$, and $K$ are constants [8]. However, it has generally been recognized that the exponential equation is poorly fitted for data including the low range of lung volumes, particularly for lungs in acute respiratory distress syndrome (ARDS). To overcome this poor fitting other equations including first or third-degree polynomials were proposed, but the increased number of parameters had little or no physiological meaning [9]. In 1998, Venegas et al. found a comprehensive sigmoid equation (the logistic equation) in dimensionless form describing PV curves in deflation and inflation with good fitting [10]. The logistic equation has been recognized to fit PV curves of a whole range sufficient even in patients with idiopathic pulmonary fibrosis (IPF) [11].

In this paper, after thermo-statistic considerations on alveolar micromechanics an entropy model of the lungs was constructed for describing the static PV relationship. By use of the logistic equation for static PV curves, the internal energy of the lungs was expressed as a set of equations with corresponding parameters of the logistic equation for the PV relationship. As the result, the relationship between the internal energy $\left(U_{L}\right)$ and its corresponding lung volume $\left(V_{L}\right)$ was constructed as the $V_{L}-U_{L}$ diagrams from reported data of parameters 
estimated by the logistic model for static PV curves during anesthesia in patients with IPF and patients with myasthenia gravis (MG). Compared with the concept of tissue energy/surface energy and the $V_{L}-U_{L}$ diagram, it was concluded that the tissue energy and the surface energy could be represented by the minimal value $U_{o}$ and the shape of the $V_{L}-U_{L}$ diagram. Since the $V_{L}-U_{L}$ diagram is easy to construct by use of the estimated parameters from the logistic equation of each static PV curve, these indicators could be useful to optimize setting the mechanical ventilation of an individual patient in the intensive care unit (ICU). To conclude, it is necessary to develop easy tools for fitting the individual sigmoid PV curve to the logistic equation in clinical situations.

\section{Assumptions}

1) Pulmonary lobules are integrated by a fractal bronchial tree as the architecture of lungs (Figure 2: based on images from [6] and [7])

Recent imaging technology including high-resolution computer assisted tomography (HRCT) of the lungs revealed that Miller's secondary pulmonary lobule is the basic unit of pulmonary parenchyma from the perspective of structural functional relationship [12]:

a) The secondary pulmonary lobule has one corresponding bronchiole, through which airflow goes inward and outward. The secondary pulmonary lobule has many alveoli and bronchioles tangled where $\mathrm{O}_{2}-\mathrm{CO}_{2}$ gas-exchange by erythrocytes occurs in the arterioles and capillary beds. The alveoli and bronchioles are anatomically interrelated and function as a unit body [13];

(a)

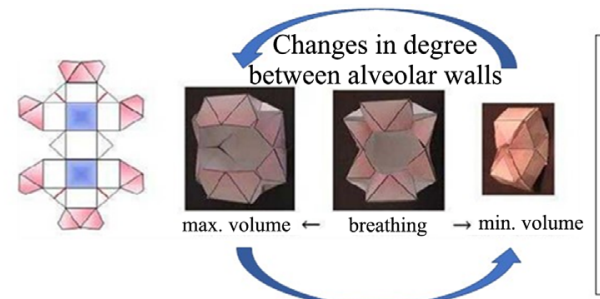

(b)

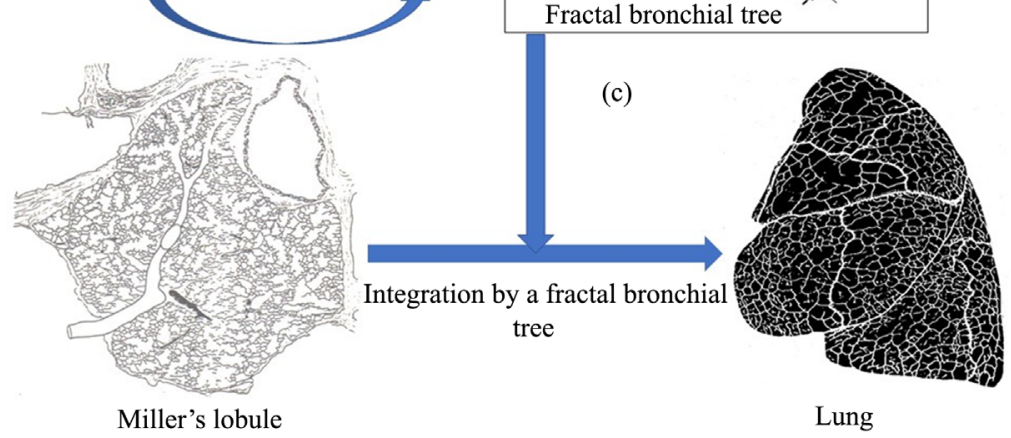

Figure 2. Pulmonary lobules and a fractal bronchial tree model. (a): The origami model for an alveolar polyhedron shows a change in volume is dependent on the angles between adjacent walls of alveolar polyhedron; (b): A secondary pulmonary lobule (Miller's lobule) has a corresponding single bronchiole and single arteriole for supplying air and blood, and has numerous alveolar polyhedrons; (c): The fractal bronchial tree (power laws are seen among the diameter and corresponding length) integrates numerous pulmonary lobules into the respiratory system as a body of organ. 
b) According to the origami (paper craft) model of the alveolar structure [6], alveolar polyhedrons are packed in the secondary pulmonary lobule, and the angle between adjacent alveolar walls changes depending on the degree of volume in a pulmonary lobule without changes in length of elastic fibers existing in alveolar walls;

c) More than approximately 3000 secondary pulmonary lobules are integrated into the whole lung by an asymmetrical branching of the bronchial tree (from lobular bronchial branch to trachea), through which lobular flows make a respiratory flow as a summation;

d) The asymmetrical branching tree of the bronchial system is defined as a "fractal tree" characterized by power laws between the corresponding diameter and length in each branch such that $r_{1}^{n}=r_{2}^{n}+r_{3}^{n}, L_{1} / r_{1}^{i}=L_{2} / r_{2}^{i}=L_{3} / r_{3}^{i}$, and $i+n=4$ for three branches at bifurcation $(n=2.7$ and $i=1.3$ for the human bronchial tree). As a result, each drop in pressure along a branch is equal in the fractal bronchial tree under a constant laminar flow [14].

2) Logistic equation of the static PV relationship of respiratory system

Analytical fittings of quasi-static pressure volume curves composed of the transpulmonary pressure $P_{t p}$ (the difference between the pleural pressure $P_{p l}$ and the occlusive pressure at the orifice of tracheal tube $P_{o}$, i.e., $\left.P_{t p}=P_{o}-P_{p l}\right)$ and its corresponding lung volume $V_{L}$ revealed a logistic expression in dimensionless form as follows:

$$
\frac{V_{L}-a}{b}=\frac{1}{1+\exp \left\{-k\left(P_{t p}-c\right)\right\}}
$$

where $a, b, c$, and $k$ are parameters fitted for each static PV curve [10]. Parameter $a$ has units of volume and corresponds to the lower asymptote volume, which approximates the physiological variable of residual volume $(a \approx R V)$. Parameter $b$, also in units of volume, corresponds to the total change in volume between the lower and upper asymptotes, which approximates the physiological variable of vital capacity $(b \approx V C)$. Parameter $k$ corresponds to a parameter for normalizing transpulmonary pressure $P_{t p}$ to dimensionless form.

\section{Method: Thermo-Statistical Model of Pulmonary Parenchyma}

1) Internal energy $U_{L}$, Entropy $S_{L}$, and Temperature $T$

The pulmonary parenchyma is composed of more than 3000 pulmonary lobules, each of which has a corresponding single bronchiole and single arteriole for supplying air and blood. Each pulmonary lobule has numerous alveolar polyhedrons. Adjacent alveolar polyhedrons are generally not independent of each other, and it has long been understood that alveolar interdependence plays an important role in the determining strain at the level of individual septa. The assumption is that alveolar interdependence would play a role in determining the probability distribution of angles between adjacent alveolar walls, and the alveo- 
lar interdependence is expressed by the surface energy function $\varepsilon$.

Suppose that $N$-bodies of secondary pulmonary lobules make the pulmonary parenchyma according to the assumption described above. Based on Kitaoka's origami model of alveolar polyhedrons [6], each pulmonary lobule has numerous alveolar walls and changes its volume as a change in the angles $(\theta: 0 \leq \theta \leq \pi)$ between adjacent alveolar walls. Due to breathing or blood perfusion to the pulmonary lobule, each angle would fluctuate around the corresponding value $\theta$. Since the number of angles in a pulmonary lobule is huge, the probability density of $\rho_{j}(\theta)$ could be described as a continuous function.

Let a surface energy field be seen as $\varepsilon_{j}$ in the pulmonary lobule $j$. The assumption is that the value of $\varepsilon_{j}(\theta)$ independently keeps the angle between adjacent alveolar walls as $\theta$ in the pulmonary lobule $j$. In this case, probability density $\rho_{j}(\theta)$ is defined in the corresponding pulmonary lobule according to statistical mechanics as follows:

$$
\rho_{j}(\theta) \propto \mathrm{e}^{-\varepsilon_{j}(\theta) / T_{j}}
$$

where $T_{j}$ is a thermo-statistical parameter describing fluctuations of angles in the pulmonary lobule $j$ [7]. Based on the origami model, which describes changes in volume through changes in the interalveolar angles, internal energy $u_{j}$ and entropy $s_{j}$ for the secondary pulmonary lobule of $j$ containing $n_{j}$ angles are introduced as follows:

$$
\begin{gathered}
u_{j}=n_{j} \int_{0}^{\pi} \varepsilon_{j}(\theta) \rho_{j}(\theta) \mathrm{d} \theta=n_{j} \cdot \bar{\varepsilon}_{j} \\
s_{j}=-\int_{0}^{\pi} \rho_{j}(\theta) \ln \rho_{j}(\theta) \mathrm{d} \theta \propto \frac{1}{T_{j}} \int_{0}^{\pi} \varepsilon(\theta) \rho_{j}(\theta) \mathrm{d} \theta=\frac{\bar{\varepsilon}_{j}}{T_{j}}
\end{gathered}
$$

where $\bar{\varepsilon}_{j}$ is the expectation value of surface energy in the corresponding pulmonary lobule of $j$ as

$$
\int_{0}^{\pi} \varepsilon(\theta) \rho_{j}(\theta) \mathrm{d} \theta=\bar{\varepsilon}_{j}
$$

The zero law of thermodynamics (if two systems are each in thermal equilibrium with a third system, they are in thermal equilibrium with each other) can define a single parameter of temperature as $T$ in the pulmonary parenchyma consisting of pulmonary lobules [15]. Thus, the internal energy $U_{L}$ and the entropy $S_{L}$ of the lungs were defined for the lung system as follows:

$$
\begin{gathered}
U_{L}=\sum_{j=1}^{N} u_{j}=\sum_{j=1}^{N} n_{j} \cdot \bar{\varepsilon}_{j} \\
T S_{L} \propto \sum_{j=1}^{N} \bar{\varepsilon}_{j}
\end{gathered}
$$

2) Helmholtz minimum free energy for the quasi-static PV relationship

The equilibrium of a thermodynamic system in a controlled temperature and volume, which is applicable to the quasi-static PV relationship, is described as $F=U_{L}-T S_{L}$ is the minimum as follows:

$$
\mathrm{d} F=\mathrm{d} U_{L}-T \mathrm{~d} S_{L}=0
$$




\section{Results}

1) Physiologically, in the static PV relationship of the respiratory system, it was described by the mechanical equilibrium among pressures (the airway pressure $P_{o}$ and the abdominal pressure $P_{a b}$ measured at closed airway) along with the components of the respiratory system, as shown in Figure 3, as follows:

$$
\begin{gathered}
P_{t p}=P_{o}-P_{p l} \\
P_{d i}=P_{p l}-P_{a b} \\
P_{o}=P_{a b}
\end{gathered}
$$

As a result, the transpulmonary pressure $P_{t p}$ is balanced with the transdiaphragmatic pressure $P_{d i}$ as follows:

$$
P_{t p}+P_{d i}=0
$$

Thus, transpulmonary pressure $P_{t p}$ changes itself along with a change in transdiaphragmatic pressure $P_{d i}$, which has zero condition as the standard condition at $\left(c, V_{o}\right)$ as follows:

$$
\begin{aligned}
& V_{o}=a+\frac{b}{2} \\
& P_{t p}\left(V_{o}\right)=c
\end{aligned}
$$

It is important to note that the elastic recoil pressure of lungs $P_{t p}$ behaves as a deflating force at more than $V_{o}$, and an inflating force at less than $V_{o}$, the same as transdiaphragmatic pressure $P_{d i}$ does. This is quite different from the classical concept for the elastic recoil pressure of the lungs, which always works as a deflating force. Then, the elastic recoil pressure of lungs $\left(P_{e l}\right)$ should be described as a difference from $c$ as follows:
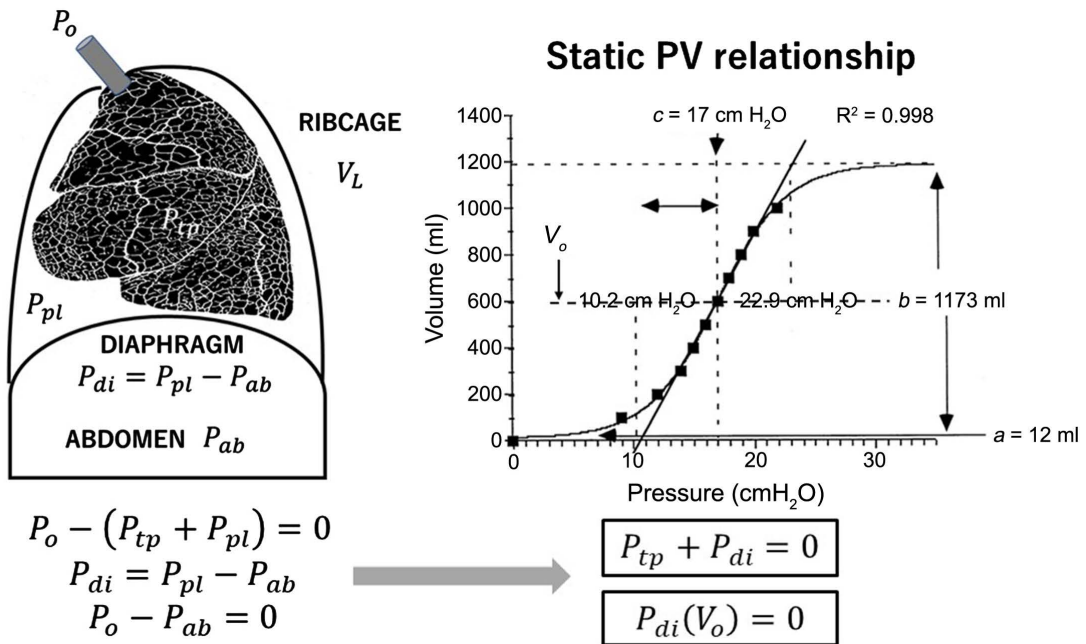

Figure 3. Mechanical balance between ribcage and lungs describing the logistic PV curve. In the static equilibrium condition, the airway opening pressure is balanced with the abdominal pressure. There is an equilibrium among components of the ribcage including the transdiaphragmatic $\left(P_{t p}\right)$ and the transpulmonary $\left(P_{d i}\right)$ pressures. It is important to note that $P_{t p}$ always balances to $P_{d i}$ (see text in detail). 


$$
P_{e l}=P_{t p}-c
$$

Therefore, the static PV relationship of the lungs (1) should be defined by the sigmoid equation (the logistic equation) as follows [9],

$$
\frac{V_{L}-a}{b}=\frac{1}{1+\exp \left\{-k P_{e l}\right\}}
$$

The first derivative of the Equation (13) was obtained as follows:

$$
\frac{\mathrm{d} V_{L}}{\mathrm{~d} P_{e l}}=k b \cdot \frac{\exp \left(-k P_{e l}\right)}{\left[1+\exp \left(-k P_{e l}\right)\right]^{2}}
$$

2) The static PV curve of the lungs requires a thermodynamic equilibrium of the Equation (8) in controlled temperature and volume as follows:

$$
\mathrm{d} U_{L}=T \mathrm{~d} S_{L}
$$

In the quasi-static state of the lungs, a change of internal energy $\mathrm{d} U_{L}$ was obtained as follows:

$$
\mathrm{d} U_{L}=-P_{e l} \mathrm{~d} V_{L}
$$

Thus, the change of pulmonary entropy $T \mathrm{~d} S_{L}$ was obtained as follows:

$$
T \mathrm{~d} S_{L}=\mathrm{d} U_{L}=-P_{e l} \mathrm{~d} V_{L}
$$

From Equations (14) and (17), $T \mathrm{~d} S_{L}$ was described as follows:

$$
T \mathrm{~d} S_{L}=-P_{e l} \mathrm{~d} V_{L}=b \cdot \frac{\left(-k P_{e l}\right) \cdot \exp \left(-k P_{e l}\right)}{\left[1+\exp \left(-k P_{e l}\right)\right]^{2}} \mathrm{~d} P_{e l}
$$

Then, a change in the internal energy of lungs $U_{L}-U_{o}$ was obtained by a definite integration of the Equation (18) in regard to $P_{e l}$ as follows (see Appendix),

$$
U_{L}-U_{o}=\int_{0}^{P_{e l}} T \mathrm{~d} S_{L} \mathrm{~d} P_{e l}=\frac{b}{k}\left[\ln \left\{\frac{2 \exp \left(-k P_{e l}\right)}{1+\exp \left(-k P_{e l}\right)}\right\}-\frac{-k P_{e l}}{1+\exp \left(-k P_{e l}\right)}\right]
$$

where $U_{o}$ is the internal energy at the standard condition of $P_{t p}=c$, at which the lung volume was obtained as $V_{o}=a+b / 2$. Then, the lung volume $V_{L}$ was expressed as the logistic equation as follows:

$$
V_{L}-V_{o}=b\left\{\frac{1}{1+\exp \left(-k P_{e l}\right)}-\frac{1}{2}\right\}
$$

3) Two basic functions $g$ and $v$ for every secondary pulmonary lobule were taken out from Equations (13) and (19) as follows:

$$
\begin{gathered}
g=\left[\ln \left\{\frac{2 \exp (-p)}{1+\exp (-p)}\right\}-\frac{(-p)}{1+\exp (-p)}\right] \\
v=\frac{1}{1+\exp (-p)}
\end{gathered}
$$

Then, parameter $p$ was obtained from the equation as follows: 


$$
-p=\frac{\mathrm{d} g}{\mathrm{~d} v}
$$

These functions were taken out as thermodynamic state functions in every secondary pulmonary lobule from self-similarity in the architecture of pulmonary segments and lobules (as described in the section of assumptions). Then, the internal energy and corresponding volume for the secondary pulmonary lobule $j$ were described as follows,

$$
\begin{aligned}
& u_{j}-u_{o j}=\frac{b_{j}}{k} g \\
& v_{j}-v_{o j}=b_{j} v
\end{aligned}
$$

where $u_{o j}$ and $v_{o j}$ are the internal energy and the volume of secondary pulmonary lobule $j$ at the standard condition, respectively. Thus, the internal energy of lung $U_{L}$, the corresponding lung volume $V_{L}$ were defined as the summation of every contributing secondary pulmonary lobules as shown in Figure 4 as follows:

$$
\begin{gathered}
U_{L}=\sum_{j=1}^{N} u_{j}=\frac{g}{k} \sum_{j=1}^{N} b_{j}+\sum_{j=1}^{N} u_{o j}=\frac{g}{k} b+U_{o} \\
V_{L}=v \sum_{j=1}^{N} b_{j}+\sum_{j=1}^{N} v_{o j}=v b+V_{o} \\
b=\sum_{j=1}^{N} b_{j} \\
P_{e l}=-\frac{\mathrm{d} U_{L}}{\mathrm{~d} V_{L}}=-\frac{\mathrm{d}\left(\frac{g b}{k}\right)}{\mathrm{d}(v b)}=-\frac{1}{k} \frac{\mathrm{d} g}{\mathrm{~d} v}=\frac{p}{k}
\end{gathered}
$$

As the result, the PV relationship of lungs composed of pulmonary secondary lobules was transformed to the volume-energy $\left(V_{L}-U_{L}\right)$ relationship by a set of equations as well as follows:

$$
\begin{gathered}
p=k\left(P_{t p}-c\right) \\
V_{o}=a+\frac{b}{2} \\
U_{o}=c V_{o}=c\left(a+\frac{b}{2}\right) \\
V_{L}-V_{o}=b\left\{\frac{1}{1+\exp (-p)}-\frac{1}{2}\right\} \\
U_{L}-U_{o}=\frac{b}{k}\left[\ln \left\{\frac{2 \exp (-p)}{1+\exp (-p)}\right\}-\frac{-p}{1+\exp (-p)}\right]
\end{gathered}
$$

4) Ferreria et al. reported the set of parameters with the sigmoid model of static PV relationship for patients with IPF and MG as shown in Table 1 [11]. A set of Equations (30), (31), (32), (33), and (34) depicted the $V_{L}-U_{L}$ relationship for each patient by using the parameters estimated in Table 1, as shown in Figure 5, where the minimum value of internal energy was represented by the bottom point of each curve $\left(U_{o}\right)$. 


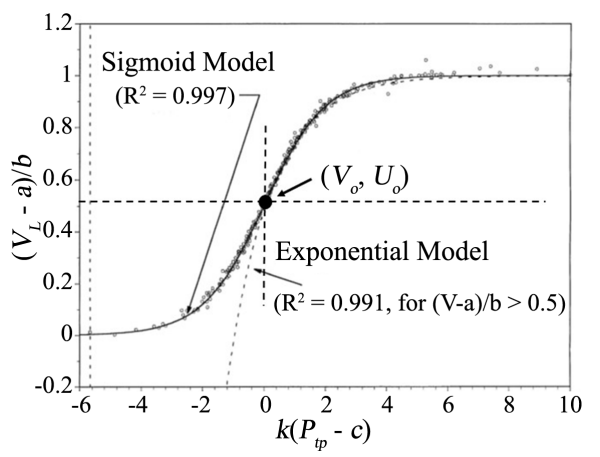

(a)

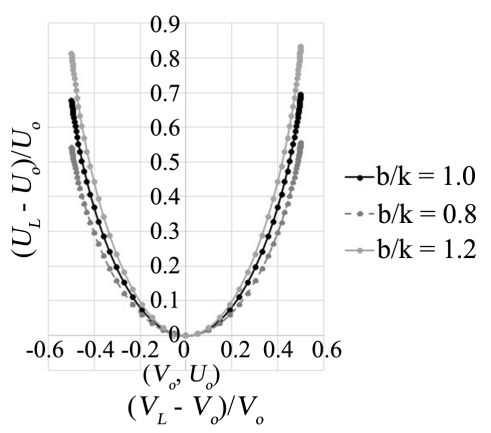

(b)

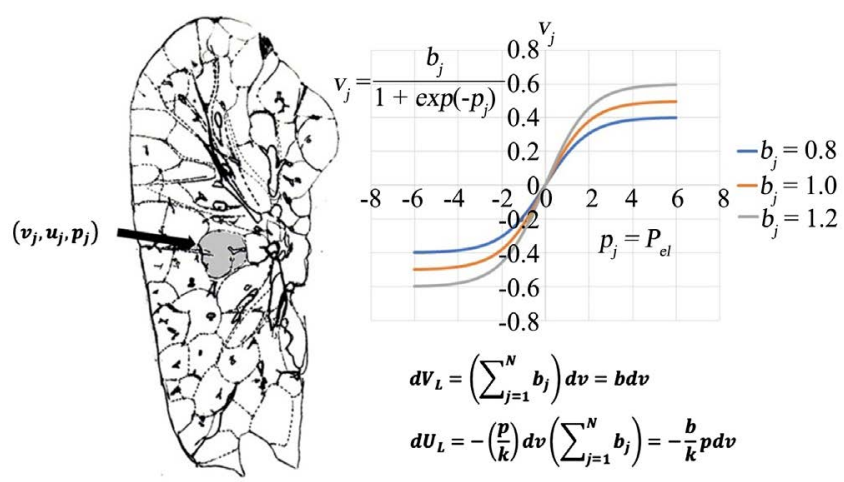

(c)

Figure 4. The sigmoid (logistic) PV relationship in dimensionless form and the volume-energy relationship. (a): Venegas et al. [10] displayed that the sigmoid PV relationship showed good fitting for PV curves, and that the exponential PV relationship showed poor fitting for PV curves in the region of volume less than $50 \%$ of the total lung capacity (TLC); (b): The volume-energy relationships constructed from the logistic PV curves were drawn in the upper right panel when $b / k$ was $0.8,1.0$, and 1.2 , respectively; (c): Based on the self-similarity in the segmental structure of lungs, each secondary pulmonary lobule was assumed to have its own logistic volume-pressure relationship. By use of the common volume-energy $(g-v)$ relationship, the volume-energy relationship of the lungs was reconstructed as a set of equations.

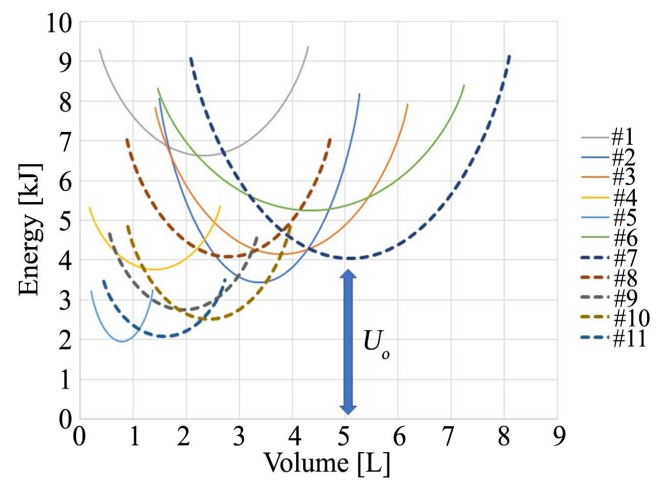

Figure 5. Constructed volume-energy diagrams from parameters reported in the paper of Ferreria et al. The individual diagram was constructed from the estimated parameters shown in Table 1 by Ferreria et al. [11] for each patient on a mechanical ventilator, which is indicated by numbers 1 to 11 . Each diagram has its own minimum value of $U_{o}$, and its own specific shape dependent on $b / k$. 
Table 1. Parameters in the logistic equation for PV curves reported by Ferreria et al. [11] and corresponding minimal energies calculated.

\begin{tabular}{cccccccc}
\hline$\#$ & Diagnosis & $\mathrm{b}(\mathrm{L})$ & Vo $(\mathrm{L})$ & $\mathrm{c}(\mathrm{kPa})$ & $1 / \mathrm{k}(\mathrm{kPa})$ & $\mathrm{Vo}(\mathrm{L})$ & Uo $(\mathrm{kJ})$ \\
\hline 1 & IPF & 3.94 & 1.61 & 2.84 & 1.01 & 2.33 & 6.62 \\
2 & IPF & 3.78 & 0.41 & 1.02 & 1.81 & 3.37 & 3.43 \\
3 & IPF & 4.78 & 0.99 & 1.10 & 1.13 & 3.79 & 4.16 \\
4 & IPF & 2.47 & 1.05 & 2.67 & 0.93 & 1.41 & 3.77 \\
5 & IPF & 1.15 & 0.36 & 2.46 & 1.63 & 0.79 & 1.95 \\
6 & IPF & 5.79 & 1.44 & 1.21 & 0.79 & 4.34 & 5.24 \\
7 & MG & 6.02 & 0.93 & 0.79 & 1.23 & 5.09 & 4.05 \\
8 & MG & 3.84 & 1.04 & 1.46 & 1.13 & 2.80 & 4.09 \\
9 & MG & 2.81 & 0.86 & 1.40 & 1.01 & 1.95 & 2.74 \\
10 & MG & 3.07 & 0.64 & 1.03 & 1.12 & 2.43 & 2.51 \\
11 & MG & 2.29 & 0.70 & 1.31 & 0.90 & 1.59 & 2.09 \\
\hline
\end{tabular}

Each value was described in MKS units of International Union of Pure and Applied Chemistry (IUPAC): $\mathrm{ml} \rightarrow \mathrm{L} ; \mathrm{cmH}_{2} \mathrm{O} \rightarrow \mathrm{kPa} ; \mathrm{cmH}_{2} \cdot \mathrm{ml} \rightarrow \mathrm{kJ}$. Numbers 1 to 11 indicate an individual patient. Patients numbered 1 to 6 have idiopathic pulmonary fibrosis (IPF), and patients numbered 7 to 11 have myasthenia gravis (MG).

\section{Discussion}

Classically static PV relationships of the respiratory system have been explained by the mechanical balance between forces in the ribcage and the lungs, where the lungs have been recognized to always behave as inflating from the residual lung volume (RV) to the total lung capacity (TLC). This property of lungs has usually been described by the exponential equation. Instead, the sigmoid (the logistic) equation applied in this study describes quite different physiological behavior of the lungs: the Equation (10) of $P_{t p}+P_{d i}=0$ explains that the lungs reduce volume at more than $V_{o}$, and expand at less than $V_{o}$.

Lung elasticity has been recognized to originate mainly from the tissue components of the lung skeleton composed of a continuous network of elastic fibers that connect the arteries, airways, and interstitial space. This component of elasticity was described by the exponential equation. However, another important component providing elasticity to the lung is the so-called surface force, generated at the interface between air and the alveolar lining layer, where surfactant is an important element. In the excised lung, elasticity is measured as the relationship between lung volume and pressure measured at the closed airway (PV curve), after the lung has inflated, as shown in Figure 5: the left panel shows the PV curve of an excised lung filled with saline solution (the solid lines), which is described by the exponential equation. This has been recognized to mainly represent the properties of the tissue components, whereas that of the gas-filled lung includes both the tissue properties and the surface tension exerted along the alveolar walls, which is described by the sigmoid or logistic equation (the dot lines). Therefore, transformation of the PV relationship from an exponential 
equation to a logistic equation through emergence of the standard condition at the point of pressure $c$ is suggested caused by mechanical effects of the surfactant system in the lung.

Static PV curves are measured several seconds after changes in the volume are made. Such curves represent the averaged mechanical properties of the whole lung. The lungs are composed of a large number of secondary pulmonary lobules with integration through the bronchial tree as described in assumption 1 . The upper limits of the air-filled curve are made by all the secondary lobules at about $30 \mathrm{cmH}_{2} \mathrm{O}$, and the lower limit of the curve is from air trapping at the lobular bronchioles. The prominent initial flat portion of the inflation curve up to about $10 \mathrm{cmH}_{2} \mathrm{O}$ in the upper panel of Figure 1) is interpreted as being the onset of recruitment of previously unventilated pulmonary lobules. The difference between curves during inflation and deflation is called "hysteresis of lungs", which is measured by the area of PV loop and described as the entropy production $(D)$ of Oliveria et al. [16] as follows:

$$
\text { Entropy production }(D)=\oint P_{e l} \mathrm{~d} V_{L}=\oint \frac{p}{k} b \mathrm{~d} v=\oint \frac{b}{k} p \mathrm{~d} v
$$

Based on the thermodynamic model in this paper, since $p$ and $v$ are thermodynamic state functions, the area of the PV loop is zero as follows:

$$
\oint p \mathrm{~d} v=0
$$

In comparing the volume-energy diagrams of deflation and inflation (Figure 5), the minimum point (black dots in Figure 5) of the inflation curve is shifted upper and its shape transformed to a slightly wide curve. The entropy production $(D)$ is represented as the area enclosed by the inflation and deflation $V_{L}$ $U_{L}$ diagrams (the dark area in Figure 6). The difference between the $V_{L}-U_{L}$ diagrams is caused by the difference in $b$, which is described as follows:

$$
b=\sum_{j=1}^{N} \sigma_{j} b_{j}
$$
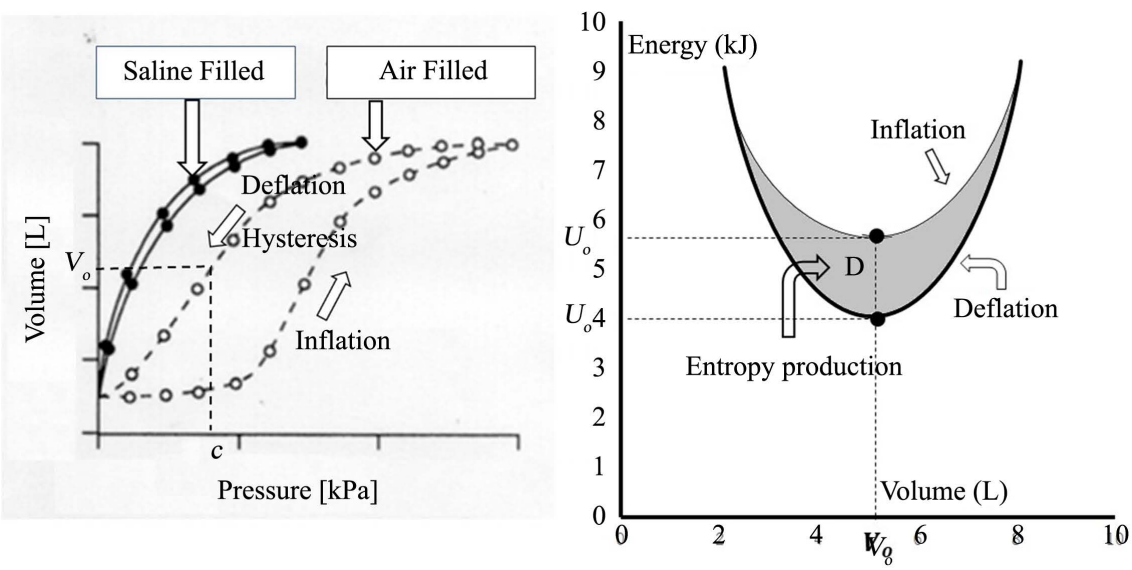

Figure 6. Hysteresis and the entropy production. Comparison of the PV relationships and the volume-energy relationship showed the entropy production of lung hysteresis quantitatively as the area of difference between volume energy diagrams. 
where $\sigma_{j}$ indicates a state of the lobular bronchiole $j$ open (1) or closed (0). That is, $D$ would relate to the number of contributing lobular bronchioles reflecting dynamic conditions of the bronchial tree modified by physiological actions of the pulmonary surfactant, which is a surface-active lipoprotein complex (phospho-lipoprotein) secreted locally from cells including type II alveolar cells and Clara cells, which are non-ciliated, non-mucous, and secretory cells, in the lobular bronchioles [17].

Fitting an exponential equation to the deflation PV curves of lungs has been applied at excluding points below $50 \%$ of the total lung capacity, and has been shown useful clinically when applied to the deflation limb of the PV curve of spontaneously breathing patients. Instead, a sigmoidal model is superior when an inflation PV curve is captured in anesthetized patients because that it is necessary to fit the lower limb of the PV curve to titrate mechanical ventilation for each patient [11]. The entropy model of each $V_{L}-U_{L}$ diagram may help to titrate mechanical ventilation accordingly in individual patient.

\section{Conclusion}

First, the author revised the mechanical balance among classical components of the chest wall and lungs during the static PV changes, resulting in finding that the transdiaphragmatic pressure always balances with the transpulmonary pressure. Through thermo-statistic considerations on alveolar micromechanics, a thermo-statistical model (entropy model) that would give a central role in the static PV relationship of air-filled lungs was obtained. The entropy model led to getting a set of thermodynamic state functions as common as the internal energy $g$ and volume $v$ in every secondary pulmonary lobule from the logistic equation for static sigmoid PV curves of the lungs. Based on reconstruction of the static PV relationship by use of $g$ and $v$, the logistic PV relationship was transformed into the volume-energy relationship defined by a set of equations. An individual volume-energy diagram was drawn by use of clinically estimated parameters of each patient on the mechanical ventilator. Thus, the tissue components and the surface component may be estimated as the minimum energy and the shape of energy function respectively in an individual patient. Furthermore, the "entropy production" of lung hysteresis may be estimated by the difference between the $U_{L}-V_{L}$ diagrams, and would describe the dynamics of the bronchial tree modified by the surfactant system. The $V_{L}-U_{L}$ diagram is easy to construct by use of estimated parameters from the logistic equation of each static sigmoid PV curve. Therefore, the $V_{L}-U_{L}$ analysis on the logistic PV curve including the entropy production might be useful to optimize setting the mechanical ventilation of individual patients in the intensive care unit (ICU). To conclude, it is necessary to develop easy tools usable in ICU for fitting the sigmoid PV curve by the logistic equation.

\section{Conflicts of Interest}

The author declares no conflicts of interest regarding the publication of this paper. 


\section{References}

[1] Grassino, A.E. and Roussos, C. (1997) Static Properties of the Lung and Chest Wall. In: Crystal, R.G., West, J.B., et al., Eds., The Lung. Scientific Foundations, 2nd Edition, Lippincott-Raven Publishers, Philadelphia, 1187-1201.

[2] Murray, J.F. (1976) The Normal Lung. WA Saunders, Philadelphia, 83.

[3] Fredberg, J.J. and Kamm, R.D. (2006) Stress Transmission in the Lung: Pathways from Organ to Molecule. Annual Review of Physiology, 68, 507-541. https://doi.org/10.1146/annurev.physiol.68.072304.114110

[4] Gil, J., Bachofen, H., Gehr, P. and Weibel, E.R. (1979) Alveolar Volume-Surface Area Relation in Air- and Saline-Filled Lungs Fixed by Vascular Perfusion. Journal of Applied Physiology, 47, 990-1001. https://doi.org/10.1152/jappl.1979.47.5.990

[5] Knudsen, L. and Ochs, M. (2018) The Micromechanics of Lung Alveoli: Structure and Function of Surfactant Tissue Components. Histochemistry and Cell Biology, 150, 661-676. https://doi.org/10.1007/s00418-018-1747-9

[6] Kitaoka, H., Hoyos, C.A. and Takaki, R. (2010) Origami Model for Breathing Alveoli. Advances in Experimental Medicine and Biology, 669, 49-52. https://doi.org/10.1007/978-1-4419-5692-7_10

[7] Min, K.Y. (2015) Entropy Change of Lungs: Determinant of the Static Properties of the Lungs. Applied Mathematics, 6, 1200-1207. https://doi.org/10.4236/am.2015.68111

[8] Salazar, E. and Knowles, J.H. (1964) An Analysis of Pressure-Volume Characteristics of the Lungs. Journal of Applied Physiology, 19, 97-104. https://doi.org/10.1152/jappl.1964.19.1.97

[9] Murphy, B.G. and Engel, L.A. (1978) Models of the Pressure-Volume Relationship of the Human Lung. Respiration Physiology, 32, 183-194. https://doi.org/10.1016/0034-5687(78)90108-1

[10] Venegas, J.G., Harris, R.S. and Simon, B.A. (1985) A Comprehensive Equation for the Pulmonary Pressure-Volume Curve. Journal of Applied Physiology, 84, 389-395. https://doi.org/10.1152/jappl.1998.84.1.389

[11] Ferreira, J.C., Bensenor, F.E.M., Rocha, M.J.J., Salge, J.M., Harris, R.S., Malhotra, A., et al. (2011) A Sigmoidal Fit for Pressure-Volume Curves of Idiopathic Pulmonary Fibrosis Patients on Mechanical Ventilation: Clinical Implications. Clinics, 66, 1157-1163. https://doi.org/10.1590/S1807-59322011000700006

[12] Webb, W.R. (2006) Thin-Section CT of the Secondary Pulmonary Lobule: Anatomy and the Image-The 2004 Fleischner Lecture. Radiology, 239, 322-338. https://doi.org/10.1148/radiol.2392041968

[13] Tabuchi, A., Styp-Rekowska, B., Slutsky, A.S., Wagnaer, P.D., Pries, A.R. and Kuebler, W.M. (2012) Precapillary Oxygenation Contributes Relevantly to Gas Exchange in the Intact Lung. American Journal of Respiratory and Critical Care Medicine, 188, 474-481. https://doi.org/10.1164/rccm.201212-2177OC

[14] Min, K.Y., Hosoi, K., Kinoshita, Y., Hara, S., Degami, H., Takada, T. and Nakamura, T. (2012) Use of Fractal Geometry to Propose a New mechanism of Airway-Parenchymal Interdependence. Open Journal of Molecular and Integrative Physiology, 2, 14-20. https://doi.org/10.4236/ojmip.2012.21003

[15] Kittel, C. and Kroemer, H. (1980) Entropy and Temperature. In: Kittel, C. and Kroemer, H., Eds., Thermal Physics, 2nd Edition, WH Freeman and Company, New Work, 27-54.

[16] Oliveira, C.L.N., Araujo, A.D., Bates, J.H.T., Andrade Jr., J.S. and Suki, B. (2016) 
Entropy Production and the Pressure-Volume Curve of the Lung. Frontiers in Physiology, 7, 73. https://doi.org/10.3389/fphys.2016.00073

[17] Kingma, P. and Jobe, A.H. (2019) The Surfactant System. In: Wilmott, R., Bush, A., Deterding, R., Ratjen, F., Sly, P., Zar, H. and Li, A., Eds., Kendig's Disorders of the Respiratory Tract in Children, 9th Edition, Elsevier, Amsterdam, 57-62. 


\section{Appendix}

When it is $f=\exp \left(-k P_{e l}\right)$, the following equation is obtained,

$$
\mathrm{d} f=-k \cdot \exp \left(-k P_{e l}\right) \mathrm{d} P_{e l}=-k f \mathrm{~d} P_{e l}
$$

Then, the Equation (12) is expressed as follows:

$$
T \mathrm{~d} S_{L}=-P_{e l} \mathrm{~d} V_{L}=-\frac{b}{k} \cdot \frac{\ln f}{[1+f]^{2}} \mathrm{~d} f
$$

Therefore, $U_{L}-U_{o}$ is expressed as follows:

$$
\begin{aligned}
U_{L}-U_{o} & =-\frac{b}{k} \int_{1}^{\exp \left(-k P_{e l}\right)} \frac{\ln f}{[1+f]^{2}} \mathrm{~d} f=-\frac{b}{k}\left[\int_{1}^{\exp \left(-k P_{e l}\right)} \ln f \cdot\left(\frac{1}{1+f}\right)^{\prime} \mathrm{d} f\right] \\
& =-\frac{b}{k}\left\{\left[\ln f \cdot \frac{1}{1+f}\right]_{1}^{\exp \left(-k P_{e l}\right)}-\int_{1}^{\exp \left(-k P_{e l}\right)}\left(\frac{1}{f} \cdot \frac{1}{1+f}\right) \mathrm{d} f\right\} \\
& =-\frac{b}{k}\left\{\left[-k P_{e l} \cdot \frac{1}{1+\exp \left(-k P_{e l}\right)}\right]-\int_{1}^{\exp \left(-k P_{e l}\right)}\left(\frac{1}{f}-\frac{1}{1+f}\right) \mathrm{d} f\right\} \\
& =-\frac{b}{k}\left\{\left[\frac{-k P_{e l}}{1+\exp \left(-k P_{e l}\right)}\right]-\left[\ln \left(\frac{f}{1+f}\right)\right]_{1}^{\exp \left(-k P_{e l}\right)}\right\} \\
& =-\frac{b}{k}\left\{\frac{-k P_{e l}}{1+\exp \left(-k P_{e l}\right)}-\ln \frac{\exp \left(-k P_{e l}\right)}{1+\exp \left(-k P_{e l}\right)}+\ln \frac{1}{2}\right\} \\
& =\frac{b}{k}\left\{\ln \left[\frac{2 \cdot \exp \left(-k P_{e l}\right)}{1+\exp \left(-k P_{e l}\right)}\right]-\frac{-k P_{e l}}{1+\exp \left(-k P_{e l}\right)}\right\}
\end{aligned}
$$

